\title{
Personalized Medicine in Urologic Oncology
}

\author{
Yair Lotan, ${ }^{\infty 1}$ Jack Schalken, ${ }^{2}$ Nathan Lawrentschuk, ${ }^{3,4}$ \\ ${ }^{1}$ Department of Urology, University of Texas Southwestern Medical Center Dallas, United States, ${ }^{2}$ Department of Urology, Radboud University Medical Center, \\ Nijmegen, The Netherlands, ${ }^{3}$ Department of Surgical Oncology, Peter MacCallum Cancer Centre, Melbourne, Australia, \\ ${ }^{4}$ Department of Urology, Royal Melbourne Hospital, Australia
}

Soc Int Urol J. 2020;1(1):6-7

In an ideal world, the diagnosis and treatment of disease would be non-invasive and precise. Biologic testing would be performed using easily available samples such as blood, urine, sputum, and skin swab, which would provide information regarding the cause of a disease as well as prognosis and optimal treatment. Although in some areas there has been progress toward these goals, in others, significant work must still be done before molecular markers can be incorporated into common practice. In 2019, the Société Internationale d'Urologie (SIU), the International Consultation on Urological Diseases (ICUD) and the World Urologic Oncology Forum (WUOF) convened key opinion leaders from around the world to review the current use of molecular markers for the diagnosis and management of urologic malignancies, as well as developments in research. This special edition of the SIUJ presents a summary of their work.

An ideal marker would fulfill strict criteria demonstrating that it provides clinical benefit, information that is independent from other clinical factors, cost-effectiveness, and reproducibility, and would be comprehensively validated. The pathway to incorporate molecular markers should be standardized, similar to that required for approval of medications. A marker should pass through several phases, as outlined by Melissa Assel and Andrew Vickers [1], in which it is tested against the clinical question that it is supposed to answer and should then be validated in multicenter prospective cohorts. Many markers do not survive the initial phase of discovery and evaluation in small retrospective cohorts. This phase, while exciting, is subject to risk of bias and overfitting. If a marker is developed that shows promise, the next phase of evaluation should involve validation in independent cohorts. Finally, a test of clinical utility should be performed on a prospective cohort in which the marker is evaluated for its ability to change clinical management to the benefit of the patient.

In this issue of the SIUJ, Ankeet Shah and colleagues discuss the sources from which most molecular biomarkers are obtained and the considerations that are associated with each [2]. Blood-based markers have had major impact in the management of testis and prostate cancer. Diagnosis and management of testis cancer includes the use of alpha-fetoprotein and beta-hCG. These markers help in differentiating histology and can guide use of chemotherapy, but they have limitations related to sensitivity and specificity. Aditya Bagrodia and colleagues examine the role of these markers and new promising markers, especially miRNA, in the management of testicular germ cell tumors [3]. Prostate specific antigen, in contrast, was developed for surveillance of patients with prostate cancer but has been incorporated into screening programs. The use of PSA for screening has resulted in decades of controversy, since evaluation of the impact of prostate cancer screening on survival occurred many years after widespread utilization of the marker for this purpose [4]. Renu Eapen and colleagues examine serum-based markers that have been developed with the intention of overcoming some of the limitations of PSA [5]. Gillian Vandekerkhove and Alexander Wyatt focus specifically on the development of circulating tumor DNA as a clinically useful biomarker for prostate cancer [6].

Urine-based markers have had the biggest impact in the management of bladder cancer. Urine cytology have been incorporated into guidelines despite poor sensitivity for low-grade cancer and moderate sensitivity for high-grade disease. Other urine markers have not been adopted because of failure to fulfill stringent criteria of performance. In this issue of the SIUJ, Tilman Todenhöfer and colleagues review the performance of currently available urine-based tumor markers for bladder cancer detection [7], and Laura-Maria Krabbe et al. give an overview of the clinical utility of these markers [8]. Similarly, for prostate cancer there are attempts to improve detection using urinary biomarkers, and these are reviewed by Renu Eapen and colleagues [5].

The utility of tissue-based markers is limited by the accessibility of tissue. Nonetheless, in specific clinical scenarios where tissue is readily available, tissue markers can be invaluable. Peter Lonergan and colleagues [9] report on exciting developments in tissue biomarkers for prostate cancer, while Liang Qu and colleagues discuss the utility of tissue markers for diagnosis and classification of renal cell carcinoma [10]. 
The emphasis on achieving a high standard of evidence before incorporating markers into clinical practice has merit [1]. It is particularly important because of the risk associated with false positive results, which often lead to unnecessary testing and anxiety. Furthermore, these tests may contribute significantly to the cost of health care, a worldwide concern. Some biomarkers have been adopted without sufficient evidence of effectiveness, resulting in controversy.

The Cancer Genome Atlas, which molecularly characterized over 11000 cancers [11], has provided important new information regarding urologic cancers, that is now being used to characterize tumor behavior, prognosis, and response to therapy. This program, along with advances in next-generation sequencing will advance the goals of personalized medicine, and significant effort is needed to interpret this information using bioinformatics and to validate its utility in clinical care. Multiple new clinical trials are based on targeting specific mutations or enriching the patient population for the expression of immunological markers such as

\section{References}

1. Assel M, Vickers AJ. SIU-ICUD Consultation on Molecular Biomarkers in Urological Oncology: Clinical development of biomarkers. Soc Int Urol J. 2020;1(1):16-22.

2. Shah A, Grimberg DC, Inman BA. SIU-ICUD Consultation on Molecular Biomarkers in Urological Oncology: Classification of molecular biomarkers. Soc Int Urol J. 2020;1(1):8-15.

3. Bagrodia A, Daneshmand S, Cheng L, Amatruda J, Murray M, Lafin JT. SIU-ICUD Consultation on Molecular Biomarkers in Urological Oncology: The past and future of biomarkers in testicular germ cell tumors. Soc Int Urol J. 2020;1(1):77-84.

4. PLCO Project Team, Gohagan JK, Prorok PC, Hayes RB, Kramer B-S. The Prostate, Lung, Colorectal and Ovarian (PLCO) Cancer Screening Trial of the National Cancer Institute: History, organization, and status. Control Clin Trials. 2000;21(6):251S-272S. https://doi. org/10.1016/S0197-2456(00)00097-0

5. Eapen R, Lonergan PE, Bagguley D, Ong S, Condon B, Meng MV. SIU-ICUD Consultation on Molecular Biomarkers in Urological Oncology: The clinical applications of serum and urinary biomarkers in prostate cancer Soc Int Urol J. 2020;1(1):30-38.

6. Vandekerkhove G, Wyatt AW. SIU-ICUD Consultation on Molecular Biomarkers in Urological Oncology: Circulating tumour DNA as a biomarker source in metastatic prostate cancer. Soc Int Urol J. 2020;1(1):39-48.
PDL1. Understanding the significance of these molecular alterations and how to use them to guide therapy will likely take years; performing trials is critical. Patients with metastatic disease, especially urothelial cancer, have a short life expectancy and do not have the luxury of being treated with multiple therapies in an attempt to find one that works. Thus, using molecular tests with predictive value to identify early the treatment with the highest likelihood of response may make a major difference for these individuals.

Rapid advances in our understanding of cancer biology are providing multiple opportunities to develop and validate molecular markers that will have a profound impact on patient management. The papers in this special issue of the SIUJ present information on currently available and potential future markers. For patients, researchers, and providers seeking to incorporate markers into practice, it is important to evaluate how well the markers perform overall and whether they can provide a tool that improves patients' care.

7. Todenhöfer T, Lodde M, van Kessel K, Pichler R, Vlahou A, Lotan Y. SIU-ICUD Consultation on Molecular Biomarkers in Urological Oncology: Urinary-based markers for bladder cancer detection. Soc Int Urol J. 2020;1(1):49-61.

8. Krabbe L-M, Gakis G, Lotan Y. SIU-ICUD Consultation on Molecular Biomarkers in Urological Oncology: Clinical utility of bladder cancer biomarkers. Soc Int Urol J. 2020;1(1):62-67.

9. Lonergan PE, Washington SL III, Meng MV, Eapen R. SIU-ICUD Consultation on Molecular Biomarkers in Urological Oncology: The clinical applications of tissue biomarkers in prostate cancer. Soc Int Urol J. 2020;1(1):23-29.

10. Qu L, Thirugnanasundralingam V, Bolton D, Finelli A, Lawrentschuk $\mathrm{N}$. Consultation on Molecular Biomarkers in Urological Oncology: Tissue-based immunohistochemical markers for diagnosis and classification of renal cell carcinoma Soc Int Urol J. 2020;1(1):68-76.

11. The Cancer Genome Atlas. The National Cancer Institute. Available from: https://www.cancer.gov/about-nci/organization/ccg/ research/structural-genomics/tcga. Accessed August 27, 2020. 